\title{
Commentary
}

\section{Explanation of the 7 Clinical Questions in Japanese Gastric Cancer Treatment Guidelines of Version 4}

\author{
Zhi Zhu, Junqing Chen* \\ Department of Surgical Oncology, the First Hospital of China Medical University, Shenyang, China \\ Email address: \\ cmucjq@126.com (Junqing Chen) \\ ${ }^{*}$ Corresponding author
}

\section{To cite this article:}

Zhi Zhu, Junqing Chen. Explanation of the 7 Clinical Questions in Japanese Gastric Cancer Treatment Guidelines of Version 4. Journal of Surgery. Vol. 6, No. 5, 2018, pp. 112-115. doi: 10.11648/j.js.20180605.11

Received: July 15, 2018; Accepted: August 8, 2018; Published: September 5, 2018

\begin{abstract}
Surgery is the main treatment for curing gastric cancer, standard D2 gastrectomy and systematical therapy provides an excellent survival outcome in East Asian countries, especially Japan, Korea, and China, however, there are many clinical questions still under debate for a long time. Japanese gastric cancer treatment guidelines has produced a great effect on the surgical treatment of gastric cancer. The treatment of gastric cancer is more standardized, rationalized and normalized. In recent years, new research results made the guideline revised. The 4th Edition of 2014 has proposed a solution for Stage IV patients with single non-curative factors. Patients with No.16a2, b1 lymphnodes metastasis, liver metastasis, or with peritoneal metastasis/CY1 disease are scheduled to receive combined treatment of operation and chemotherapy. The 4th edition incorporated new evidence that includes those delivered as a quick bulletin in the website of the Japan Gastric Cancer Association after publication of the previous version. It remains largely conformed to the textbook style, but a new section has summarized 7 clinical questions to address some important clinical issues for which hard evidence is unavailable. These questions makes the basic principles for the treatment of gastric cancer and the concept more scientific and accurate. It will provide important guidance for the future clinical practice. We provided additional comments and deeply interpreted the questions proposed by the guideline according to some new research and our clinical experience.
\end{abstract}

Keywords: Gastric Carcinoma, No.16a2, b1 Lymph Node, Peritoneal Cytology Positive, Chemotherapy

\section{Instruction}

Gastric cancer remains a major health problem around the world, despite declined incidence in recent decades. Surgery is the most efficient modality to treat gastric carcinoma. However, gastric cancer has a high recurrence rate after operation, especially in advanced stages. Adjuvant therapy with chemotherapy, chemoradiotherapy, or perioperative chemotherapy can provide survival benefit. The Japanese Gastric Cancer Treatment Guidelines committee nominated several working groups, each assigned to make relevant contributions to draw up the strategy of clinical treatment and unsolved issues in the 4th Edition. The clinical importance of lymphadenectomy and treatment of resectable metastatic cancer prompted the committee to establish a new section, a $\mathrm{Q}$ and A section was established to provide tentative best answers based on the latest envidence, whereas further discussion was deemed necessary for these topics.

\section{Commentary}

\subsection{Therapeutic Choice of Patients with Gastric Cancer When Metastasis to the Para-Aortic Lymph Nodes}

A multidisciplinary approach including surgery with para-aortic lymph node dissection could be proposed when para-aortic lymph node metastases are confined to the No. 16 a2, b1 region, provided other non-curative factors are absent.

Lymph node metastasis from gastric cancer can be classified into two types: large fusion nodules and small solitary nodules type. Large nodular lymph nodes are enlarged with the size of more than $3 \mathrm{~cm}$. The gastric cancer with large metastatic nodular lymph nodes always present few number of 
positive metastatic nodes or low metastatic lymph node ratio, localized advanced cancer, Lauren intestinal type and better prognosis. Small nodule types showed opposite biological characteristics of large nodules, diffuse type of growth pattern, it is hardly to perform radically gastrectomy, usually indicate worse prognosis.

The para-aortic lymph node metastases are confined to the No. 16 a2, b1 region, which is considered to be the large nodular type. This type is associated with better clinicopathological features. It is the pathological basis of surgical indications and play an important role in curative treatment. D2 plus para-aortic lymph node dissection (PAND) after neoadjuvant chemotherapy is the favorite surgical procedure of gastric cancer $[1,2]$. Moreover, PAND had been attempted in Japanese clinical studies until its survival benefit was denied in a randomized trial in which only patients without lymphadenopathy in the para-aortic region were eligible [3].

\subsection{Treatment of Gastric Cancer with Hepatic Metastases}

A multidisciplinary approach including surgery with curative intent could be proposed when the number of metastatic nodules is small, provided other non-curative factors are absent

The incidence of hepatic metastasis from gastric cancer is around $4-14 \%$. It is always found as multiple lesions distributed to the whole hepatic lobes and are likely to be accompanied with metastatic lesions outside of the liver. There is no effective perioperative treatment for these patients, the liver metastasis lesions could not be resected radically, the prognosis was poor. Recent years, a variety of imaging technologies have made great progress, such as ultrasonography, enhanced CT, MRI, and PET-CT. The site, number, and size of metastases can be precisely diagnosed. Many studies reported few number of metastatic nodules was regarded as a favorable prognostic factor, 5-year survival rates ranging from 10 to $40 \%$. Hepatectomy may be considered for patients with solitary metastasis or a small number of metastatic nodules with the size less than $5 \mathrm{~cm}$, and there is no other non-curative factors [4]. Adjuvant chemotherapy was recommended for the population, long-term survival was presented in highly selected patients [5]. However, there is no evidence of clinical trial on which chemotherapeutic regimen is preferred.

\subsection{Therapeutic Proposal of Gastric Cancer with Positive Peritoneal Cytology (CY1)}

Multidisciplinary treatment including standard gastrectomy can be proposed for patients with no other non-curative factors. If the CY1 status was revealed after surgery, postoperative treatment with $\mathrm{S}-1$ can be recommended as the tentative standard.

Positive peritoneal cytology CY (+) identified with cancer residual R1 is classified as stage IV in TNM staging which is equivalent to peritoneal metastasis $(+)$ with poor prognosis. CY1 patients are often performed by standard D2 gastrectomy followed by S-1 monotherapy in the absence of other no-curative factors [6]. JCOG0301 proved the procedure are more benefit than the patients who were treated with surgery alone. Recently, whether perioperative treatment is suitable may be acquire better outcomes continue to be controversial. Some clinical trials have been studied to explore neoadjuvant chemotherapy in the standard treatment [7]. However, details of the optimal multidisciplinary treatment strategy in this procedure, including the chemotherapeutic administration, chemotherapeutic regimen, and number of cycles remain to be elucidated in future clinical trials.

\subsection{Recommended Chemotherapeutic Regimen When Recurrence Was Detected Within 6 Months from Completion of the Postoperative Adjuvant Chemotherapy with $S-1$}

Although no evidence to recommend any particular regimen exists, most physicians would avoid monotherapy with S-1 for second-line chemotherapy.

Many new drugs and regimens have developed for the treatment of gastric cancer in decades, such as S-1, paclitaxel, oxaliplatin, and Cp-11. Eastern and Western scholars have put forward several well-known regimens for the treatment of gastric cancer, such as the Japanese ACTS-GC, the evidence of clinical trial of perioperative chemotherapy regimens provided that patients who received S-1 treatments survived longer after recurrence than those who did not receive S-1, regardless of the time interval between the adjuvant chemotherapy and recurrence [8]. For the recurrence within 6 months from completion of the postoperative adjuvant chemotherapy with S-1, we draw on the therapeutic experience of colorectal cancer, patients with late recurrence after adjuvant treatment have been deemed eligible for clinical trials exploring a first-line treatment, whereas those with early recurrences were considered for the second-line treatment [9]. Thus, patients with recurrences during or early after completion of the adjuvant treatment are considered as targets of second-line treatments, and S-1 monotherapy is usually avoided for this population. However, there is currently no evidence to recommend any specific regimen for this setting.

\subsection{Recommended Chemotherapeutic Regimen for Patients Suffering from Either Bowel Obstruction or Massive Ascites Due to Severe Peritoneal Metastases}

The indication for chemotherapy itself should be decided discreetly, taking into consideration the general status of the patient. Drugs with mild toxicity profiles such as infusional 5-fluorouracil and paclitaxel could be considered as the candidates.

It is hard to choose strategy for clinical doctors to face up with the patients suffering from either bowel obstruction or massive ascites due to severe peritoneal metastases.

The JCOG0106 study was designed for patients with peritoneal metastases with sequential therapy combining methotrexate plus 5FU, which had been considered promising in this procedure, was explored with continuous intravenous 
administration of 5FU as a control, but failed to show a survival benefit, while 5FU was found to be less toxic [10]. Paclitaxel was associated with a more favorable toxicity profile [11]. Researches offered some advice in selection and application of 5-FU and paclitaxel for treatment of these two cases which are very hard to cure. It isn't a standard therapy, but they provide recommendations with progressive evidence. Meanwhile, the general status of the patient should be obeseve carefully and evaluated strictly by the doctors during the treatment to avoid significant toxic reactions. The chemotherapeutic regimens above are administered on the basis of a combination of the best supportive therapies.

\subsection{Recommended Chemotherapeutic Regimen for Elderly Patients with Unresectable/Advanced Gastric Cancer}

S-1 and cisplatin could be recommended for fit patients, but utmost care should be taken since the elderly patients are generally vulnerable to the adverse events. S-1 monotherapy could be selected for more frail patients

This guideline makes a meaningful analysis and comparison on the age differences, indications, curative effect and side effects of combined chemotherapy for elderly patients with unresectable or advanced gastric cancer. There was few difference in survival between patients treated by S-1 plus cisplatin and those treated by S-1 monotherapy in a retrospective study of the elderly population of $\geqq 70$ years of age [12]. In order to improve the application of combined chemotherapy or s-1 single drug therapy in elderly patients, further evidence through clinical trials is required for various decision-makings when treating elderly patients with gastric cancer. Currenthly, the regimen of S-1 plus cisplatin will have to be decided on a patient-by-patient basis based on the experience of each physician.

\subsection{Second-Line Chemotherapeutic Regimen for HER2-Positive Gastric Cancer}

The taxanes or irinotecan can be recommended as in the case of HER2-negative cancer. However, in case a trastuzumab-containing regimen was not given as a firstline treatment, a combination of weekly paclitaxel and trastuzumab could be selected.

There is currently no evidence in support of efficacy or safety for for the specific cohort of HER2-positive patients who progressed during or after the trastuzumab-containing regimen. Either the taxanes or irinotecan could be selected as in the case of second-line treatment for HER2-negative gastric cancer. JFMC45-1102 trial reported a favoriate response rate and disease control rate of HER2-positive gastric cancer who were pretreated with paclitaxel regimen that did not contain trastuzumab [13]. Further clinical research is required for the specific regimen contained trastuzumab.

\section{Conclusions and Recommendations}

Gastrectomy with modified D2 dissection is the acceptable standard treatment worldwide now, but needs to be performed in high-volume centers by experienced surgeons. Adjuvant or perioperative chemotherapy can lower the recurrence rate and provide survival benefit. In patients with unresectable gastric cancer, chemotherapy and target therapy should be considered to improve survival and delay the appearance of disease-related symptoms. Some selective targeted agents have been developed in recent years. The only one showing strong evidence for improved survival is trastuzumab for patients with HER2-neu overexpression advanced gastric or esophagogastric junction adenocarcinoma.

\section{References}

[1] Sasako M, Sano T, Yamamoto S, Kurokawa Y, Nashimoto A, Kurita A, et al. D2 lymphadenectomy alone or with para-aortic nodal dissection for gastric cancer. The New England journal of medicine. 2008; 359:453-62.

[2] Tsuburaya A, Mizusawa J, Tanaka Y, Fukushima N, Nashimoto A, Sasako M, et al. Neoadjuvant chemotherapy with S-1 and cisplatin followed by D2 gastrectomy with para-aortic lymph node dissection for gastric cancer with extensive lymph node metastasis. The British journal of surgery. 2014; 101:653-60.

[3] Svatek RS, Dinney CP. Words of wisdom. Re: D2 lymphadenectomy alone or with para-aortic nodal dissection for gastric cancer. European urology. 2009; 56:745-6.

[4] Sakamoto Y, Sano T, Shimada K, Esaki M, Saka M, Fukagawa $\mathrm{T}$, et al. Favorable indications for hepatectomy in patients with liver metastasis from gastric cancer. Journal of surgical oncology. 2007; 95:534-9.

[5] Takemura N, Saiura A, Koga R, Arita J, Yoshioka R, Ono Y, et al. Long-term outcomes after surgical resection for gastric cancer liver metastasis: an analysis of 64 macroscopically complete resections. Langenbeck's archives of surgery. 2012; 397:951-7.

[6] Kodera Y, Ito S, Mochizuki Y, Ohashi N, Tanaka C, Kobayashi $\mathrm{D}$, et al. Long-term follow up of patients who were positive for peritoneal lavage cytology: final report from the CCOG0301 study. Gastric cancer: official journal of the International Gastric Cancer Association and the Japanese Gastric Cancer Association. 2012; 15:335-7.

[7] Mezhir JJ, Shah MA, Jacks LM, Brennan MF, Coit DG, Strong VE. Positive peritoneal cytology in patients with gastric cancer: natural history and outcome of 291 patients. Indian journal of surgical oncology. 2011; 2:16-23.

[8] Kinoshita T, Sasako M, Sano T, Katai H, Furukawa H, Tsuburaya A, et al. Phase II trial of S-1 for neoadjuvant chemotherapy against scirrhous gastric cancer (JCOG 0002). Gastric cancer: official journal of the International Gastric Cancer Association and the Japanese Gastric Cancer Association. 2009; 12:37-42.

[9] Shirao K, Boku N, Yamada Y, Yamaguchi K, Doi T, Goto M, et al. Randomized Phase III study of 5-fluorouracil continuous infusion vs. sequential methotrexate and 5-fluorouracil therapy in far advanced gastric cancer with peritoneal metastasis (JCOG0106). Japanese journal of clinical oncology. 2013; 43:972-80. 
[10] Nishina T, Boku N, Gotoh M, Shimada Y, Hamamoto Y, Yasui $\mathrm{H}$, et al. Randomized phase II study of second-line chemotherapy with the best available 5-fluorouracil regimen versus weekly administration of paclitaxel in far advanced gastric cancer with severe peritoneal metastases refractory to 5-fluorouracil-containing regimens (JCOG0407). Gastric cancer: official journal of the International Gastric Cancer Association and the Japanese Gastric Cancer Association. 2016; 19:902-10.

[11] Okabe H, Ueda S, Obama K, Hosogi H, Sakai Y. Induction chemotherapy with S-1 plus cisplatin followed by surgery for treatment of gastric cancer with peritoneal dissemination. Annals of surgical oncology. 2009; 16:3227-36.
[12] Tsushima T, Hironaka S, Boku N, Machida N, Yamazaki K, Yasui $\mathrm{H}$, et al. Comparison of safety and efficacy of S-1 monotherapy and S-1 plus cisplatin therapy in elderly patients with advanced gastric cancer. International journal of clinical oncology. 2013; 18:10-6.

[13] Nishikawa K, Takahashi T, Takaishi H, Miki A, Noshiro H, Yoshikawa T, et al. Phase II study of the effectiveness and safety of trastuzumab and paclitaxel for taxane- and trastuzumab-naive patients with HER2-positive, previously treated, advanced, or recurrent gastric cancer (JFMC45-1102). International journal of cancer. 2017; 140:188-96. 